\title{
Dynamical Behaviour in Two Prey-Predator System with Persistence
}

\author{
V. Madhusudanan ${ }^{1, a *}$ and S. Vijaya ${ }^{2, b}$ \\ ${ }^{1}$ Department of Mathematics, S.A. Engineering College, Chennai-600 077, Tamilnadu, India. \\ ${ }^{2}$ Department of Mathematics, Annamalai University, Annamalainagar, Tamilnadu, India. \\ amvms.maths@gmail.com, bhavenksho@gmail.com.
}

Keywords: Functional response, Harvesting, Local and Global stability, Persistence.

\begin{abstract}
In this work, the dynamical behavior of the system with two preys and one predator population is investigated. The predator exhibits a Holling type II response to one prey which is harvested and a Beddington-DeAngelis functional response to the other prey. The boundedness of the system is analyzed. We examine the occurrence of positive equilibrium points and stability of the system at those points. At trivial equilibrium $E_{0}$ and axial equilibrium $\left(E_{1}\right)$, the system is found to be unstable. Also we obtain the necessary and sufficient conditions for existence of interior equilibrium point $\left(E_{6}\right)$ and local and global stability of the system at the interior equilibrium $\left(E_{6}\right)$. Depending upon the existence of limit cycle, the persistence condition is established for the system. The numerical simulation infer that varying the parameters such as $e$ and $\lambda_{1}$ it is possible to change the dynamical behavior of the system from limit cycle to stable spiral. It is also observed that the harvesting rate plays a crucial role in stabilizing the system.
\end{abstract}

\section{Introduction}

Mathematical modeling for interaction between species using differential equation is one of the most classical applications to biology. Analytical techniques with computer power paved a way for better understanding and development of these models. Prey-predator models are relatively well-studied example of interactions. The dynamic relationship between predators and their prey has long been and will continue to be one of the dominant themes in mathematical ecology, due to its universal existence and importance. The most noteworthy component in prey-predator models is the "predator's functional response on prey population", it describes the amount of prey consumed by an average predator. The stability of prey-predator systems with such functional response has been the area of concentration for many theorists and experimentalists.

Two species models with functional responses are extensively studied in ecological literature [2, 9, $10,14]$. Interactions on two species continuous time systems with a predator and a prey limited only to equilibrium point or to a limit cycle. Several ecological circumstances have been analyzed by interaction between two or more species. The system representing the interaction between three species shows complex dynamical behavior $[3,4,6,7,8,12]$. The interaction of species involving persistence and extinction have been the area of interest for researchers $[1,5,11,13]$.

This paper is organized as follows. We start in section 2 by defining the mathematical model of three species population which consists of two preys and one predator. The non linear system of differential equations governed this system is introduced. Section 3 deals with the determination of equilibrium points and their existence conditions. In section 4 , we analyzed dynamical behavior of these equilibrium points. Global stability and persistence of the system is studied in section 5 . In section 6 to deals with Numerical simulation and discuss the problem.

\section{Mathematical Model}

This mathematical model consists of two prey and one predator species. One of the prey species is harvested. The predator exhibits a Holling type II response to one prey and a Beddington-DeAngelis 
functional response to the other. It is also assumed that there is no interaction between the two preys. The two prey species grow logistically and direct competition is considered between them. The mathematical model for the food web is given by the following system of equations

$$
\begin{aligned}
\frac{d X}{d t} & =r X\left(1-\frac{X}{K}\right)-\frac{a X Z}{1+b X}-H X \\
\frac{d Y}{d t} & =s Y\left(1-\frac{Y}{L}\right)-\frac{c Y Z}{1+d_{1} Y+d_{2} Z} \\
\frac{d Z}{d t} & =\frac{\lambda_{1} a X Z}{1+b X}+\frac{\lambda_{2} c Y Z}{1+d_{1} Y+d_{2} Z}-e Z
\end{aligned}
$$

Where $r, s$ capita intrinsic growth rate for preys $X$ and $Y, K$ and $L$ are carrying capacities for preys $X, Y$. Also $a$ and $c$ are capturing rates of predator $Z$ on $X$ and $Y \cdot b / a$ and $d_{1} / c$ are the predators handling time on preys $X$ and $Y \cdot H$ is the constant effort harvesting rate of prey $X, e$ is the natural death rate of predator $Z \cdot d_{2}$ measures the effect of anti-predator behavior of prey $Y \cdot \lambda_{1}$ and $\lambda_{2}$ are coefficients which measures the predators efficiency to convert prey biomass of $X$ and $Y$ respectively. We non-dimensionalize the system (1) using $x=b X, y=d_{1} Y, z=d_{2} Z$ and we obtain the following system

$$
\begin{aligned}
& \frac{d x}{d t}=r x\left[\left(1-\alpha_{1} x\right)-\frac{\beta_{1} \cdot z}{1+x}-h\right] \\
& \frac{d y}{d t}=s y\left[\left(1-\alpha_{2} y\right)-\frac{\beta_{2} \cdot z}{1+y+z}\right] \\
& \frac{d z}{d t}=e z\left[\frac{\gamma_{1} x}{1+x}+\frac{\gamma_{2} y}{1+y+z}-1\right]
\end{aligned}
$$

Where

$\alpha_{1}=1 / K b_{1}, \alpha_{2}=1 / L d_{1}, \beta_{1}=a / r d_{2}, \beta_{2}=c / s d_{2}, \gamma_{1}=\lambda_{1} a / e b, \gamma_{2}=\lambda_{2} c / e d_{1}, h=H / r$

The system (2) has to be analyzed with the following initial condition $x(0) \geq 0, y(0) \geq 0, z(0) \geq 0$.

\section{Existence of Equilibrium Points}

It can be checked that the system (2) has seven non-negative equilibrium points and three of them namely $E_{0}(0,0,0), E_{1}\left(\frac{1-h}{\alpha_{1}} 0,0\right), E_{2}\left(0, \frac{1}{\alpha_{2}}, 0\right)$ always exists. We show that the existence of other equilibrium as follows

Existence of $E_{3}(\tilde{x}, \tilde{y}, 0)$

Here $\tilde{x}, \tilde{y}$ are the positive solutions of the following algebraic equations

$$
\begin{aligned}
& r x\left(1-\alpha_{1} x-h\right)=0 \\
& s y\left(1-\alpha_{2} y\right)=0
\end{aligned}
$$

Solving (3) and (4) we get

$$
\tilde{x}=\frac{1-h}{\alpha_{1}}, \tilde{y}=\frac{1}{\alpha_{2}}
$$

Thus the equilibrium

$$
E_{3}(\tilde{x}, \tilde{y}, 0) \text { exists if } h<1, \alpha_{1}>0, \alpha_{2}>0
$$


Existence of $E_{4}(\bar{x}, 0, \bar{z})$

Here $\bar{x}, \bar{z}$ are the positive solutions of the following algebraic equations

$$
\begin{aligned}
& \left.r x\left(1-\alpha_{1} x-\frac{\beta_{1} z}{1+x}-h\right)\right)=0 \\
& e z\left(-1+\frac{\gamma_{1}}{1+x}\right)=0
\end{aligned}
$$

Solving (7) and (8) we get

$$
\bar{x}=\frac{1}{\gamma_{1}-1}, \bar{z}=\frac{\gamma_{1}}{\left(\gamma_{1}-1\right)^{2} \beta_{1}}\left[(1-h)\left(\gamma_{1}-1\right)-\alpha_{1}\right]
$$

Thus the equilibrium

$$
E_{4}(\bar{x}, 0, \bar{z}) \text { exists if } \gamma_{1}>1,(1-h)\left(\gamma_{1}-1\right)>\alpha_{1}
$$

Existence of $E_{5}(0, \hat{y}, \hat{z})$

Here $\hat{y}, \hat{z}$ are the positive solution of the following algebraic equations

$$
\begin{aligned}
s y\left(1-\alpha_{2} y-\frac{\beta_{2} z}{1+y+z}\right) & =0 \\
e z\left(-1+\frac{\gamma_{2} y}{1+y+z}\right) & =0
\end{aligned}
$$

Solving (11) and (12) we get

$$
\hat{y}=\frac{-\left(\beta_{2} \gamma_{2}-\gamma_{2}-\beta_{2}\right) \pm \sqrt{\left(\beta_{2} \gamma_{2}-\gamma_{2}-\beta_{2}\right)^{2}+4 \beta_{2} \gamma_{2} \alpha_{2}}}{2 \gamma_{2} \alpha_{2}}, \hat{z}=\hat{y}\left(\gamma_{2}-1\right)-1
$$

Thus the equilibrium

$$
E_{5}(0, \hat{y}, \hat{z}) \text { exists if } \gamma_{2}>1
$$

Existence of $E_{6}\left(x^{*}, y^{*}, z^{*}\right)$

Here $\left(x^{*}, y^{*}, z^{*}\right)$ is the positive solution of the system of algebraic equation given below:

$$
\begin{aligned}
r x\left[\left(1-\alpha_{1} x\right)-\frac{\beta_{1} \cdot z}{1+x}-h\right] & =0 \\
s y\left[\left(1-\alpha_{2} y\right)-\frac{\beta_{2} \cdot z}{1+y+z}\right] & =0 \\
e z\left[\frac{\gamma_{1} x}{1+x}+\frac{\gamma_{2} y}{1+y+z}-1\right] & =0
\end{aligned}
$$

Eliminating $z$ from (15), (16) and (17), we get

$$
f(x, y)=0 \text { and } g(x, y)=0
$$

Where

$$
\begin{aligned}
& f(x, y)=\gamma_{2} y(1+x)\left[\left(\beta_{2}-\left(1-\alpha_{2} y\right)\right]-(1+y)\left[\beta_{2}\left(1+x-\gamma_{1} x\right)\right]\right) \\
& \left.g(x, y)=\left(1-\alpha_{1} x-h\right)\left[\beta_{2}\left(1+x-\gamma_{1} x\right)\right]\right)-\beta_{1}\left[\gamma_{2} y\left(1-\alpha_{2} y\right)\right]
\end{aligned}
$$


From (19) as $x \rightarrow 0, y \rightarrow y_{a}$ is given by

$y_{a}=\frac{-B_{1}+\sqrt{B_{1}^{2}-4 A_{1} C_{1}}}{2 A_{1}}$

Where

$A_{1}=\gamma_{2} \alpha_{2}$

$B_{1}=\gamma_{2} \beta_{2}-\gamma_{2}-\beta_{2}$

$C_{1}=-\beta_{2}$

Note that $y_{a}$ is positive and real if $\beta_{2}<0$

Also from equation (19), we have $\frac{d y}{d x}=\frac{P_{1}}{Q_{1}}$

Where

$$
\begin{aligned}
P_{1} & =\gamma_{1} \gamma_{2} y\left(\beta_{2}-1-\alpha_{2} y\right) \\
Q_{1} & =\gamma_{2}(1+x)\left(\beta_{2}-1+\alpha_{2} y\right)-\beta_{2}\left(1+x\left(1-\gamma_{1}\right)\right)\left(\beta_{2}-\alpha_{2}\right)
\end{aligned}
$$

It is clear that if $P_{1}>0$ and $Q_{1}<0$, it requires $\gamma_{1}>1$ and $\beta_{2}>\alpha_{2}$

From (20) as $x \rightarrow 0, y \rightarrow y_{b}$ is given by

$$
y_{b}=\frac{-B_{2}+\sqrt{B_{2}^{2}-4 A_{2} C_{2}}}{2 A_{2}}
$$

Where

$A_{2}=\beta_{1} \gamma_{2} \alpha_{2}$

$B_{2}=-\gamma_{2}$

$C_{2}=\beta_{2}(1-h)$

Note that $y_{b}$ is positive and real if $h>1$

We also have

$\frac{d y}{d x}=-\frac{\frac{\partial g}{\partial x}}{\frac{\partial g}{\partial y}}$

We note that

$\frac{d y}{d x}<0$ if $\frac{\partial g}{\partial y}<0, \frac{\partial g}{\partial x}<0$ holds good.

Knowing the value of $x^{*}, y^{*}$, the value of $z^{*}$ can be evaluated from

$$
z^{*}=\frac{\gamma_{2} y^{*}\left(1+x^{*}\right)\left(1-\alpha_{2} y^{*}\right)}{\beta_{2}\left(1+x^{*}\left(1-\gamma_{1}\right)\right)}
$$

\section{Boundedness}

Theorem 1. The solutions $x(t), y(t)$ and $z(t)$ of system (2) initiating in $R_{+}^{3}$ are positive and bounded for all $t \geq 0$.

Proof. Since the densities of population can never be negative, obviously the solutions $x(t), y(t)$ and $z(t)$ are positive for all $t \geq 0$.

From the first equation of model (2), we have

$\frac{d x}{d t} \leq r x\left(1-\alpha_{1} x\right)$ 
By smplifying, we have

$$
\lim _{t \rightarrow \infty} \sup x(t) \leq \frac{1}{\alpha_{1}}
$$

Similarly, from the second equation of model (2)

$$
\lim _{t \rightarrow \infty} \sup y(t) \leq \frac{1}{\alpha_{2}}
$$

Consider $\Omega(t)=p_{1} x(t)+p_{2} y(t)+z(t)$. For real positive number $\eta$,

$$
\frac{d \Omega}{d t}+\eta \Omega(t)=p_{1} \frac{d x}{d t}+p_{2} \frac{d y}{d t}+\frac{d z}{d t}+\eta\left(p_{1} x(t)+p_{2} y(t)+z(t)\right)
$$

Choosing $p_{1}=\frac{e \gamma_{1}}{r \beta_{1}}, p_{2}=\frac{e \gamma_{2}}{s \beta_{2}}$ and substituting equation (2) in (26) and simplifying, we get $\frac{d \Omega}{d t}+\eta \Omega(t)=p_{1} x(r+\eta)+p_{2} y(s+\eta)-p_{1} r \alpha_{1} x_{1}^{2}-p_{2} s \alpha_{2} y^{2}-p_{1} r h x+(\eta-e) z$

If we choose $\eta \leq e$, then $\frac{d \Omega}{d t}+\eta \Omega(t) \leq p_{1}(r+\eta)+p_{2}(s+\eta)$

$$
\leq \delta
$$

Applying Birkoff Lemma on differential inequality we get,

$0 \leq \Omega(x, y, z) \leq \frac{\delta}{\eta}\left(1-e^{-\eta t}\right)+\frac{\Omega(x(0), y(0), z(0))}{e^{\eta t}}$

And for $t \rightarrow \infty, 0 \leq \Omega \leq \frac{\delta}{\eta}$

Thus all solutions of system (2) enter into the region

$B=\left\{(x, y, z): 0 \leq x \leq \frac{1}{\alpha_{1}}, 0 \leq y \leq \frac{1}{\alpha_{2}}, 0 \leq \Omega \leq \frac{\delta}{\eta}+\epsilon\right.$ for any $\left.\epsilon>0\right\}$

\section{Stability Analysis}

We shall examine the stability of the system (2), the variational matrix relating to every equilibrium steady state is measured.

$$
E(x, y, z)=\left[\begin{array}{ccc}
r-2 \alpha_{1} r x-\frac{\beta_{1} r z}{(1+x)^{2}}-h r & 0 & \frac{-\beta_{1} r x}{1+x} \\
0 & s-2 \alpha_{2} s y-\frac{\beta_{2} s z(1+z)}{(1+y+z)^{2}} & \frac{-\beta_{2} s y(1+y)}{(1+y+z)^{2}} \\
\frac{\gamma_{1} e z}{(1+x)^{2}} & \frac{\gamma_{2} e z(1+z)}{(1+y+z)^{2}} & -e+\frac{\gamma_{1} e x}{(1+x)}+\frac{\gamma_{2} e y(1+y)}{(1+y+z)^{2}}
\end{array}\right]
$$

Theorem 2. The trivial equilibrium point $E_{0}$ is stable in $z$ direction and unstable in $x-y$ direction if $h<1$. Otherwise it is stable in $x-z$ direction and unstable in $y$ direction if $h>1$.

Proof. The variational matrix for the equilibrium point at $E_{0}(0,0,0)$ is

$$
E_{0}=\left[\begin{array}{ccc}
r-h r & 0 & 0 \\
0 & s & 0 \\
0 & 0 & -e
\end{array}\right]
$$

The eigen values of $E_{0}$ are $\lambda_{1}=r-h r, \lambda_{2}=s$ and $\lambda_{3}=-e$.

If $h<1$, clearly two of the eigen vales are positive and one will be negative. In this case it is unstable manifold in $x-y$ direction and stable in $z$ direction. Suppose if $h>1$ it is stable in $x-z$ direction and unstable in $y$ direction.

This completes the proof. 
Theorem 3. The equilibrium point $E_{1}$ is stable manifold in $x-z$ direction and unstable in $y$ direction if $h<1$ and $0<\gamma_{1}<1$.

Proof. The variational matrix for the equilibrium point at $E_{1}\left(\frac{1-h}{\alpha_{1}}, 0,0\right)$ is

$$
E_{1}=\left[\begin{array}{ccc}
r(h-1) & 0 & \frac{\beta_{1} r(h-1)}{\alpha_{1}-(h-1)} \\
0 & s & 0 \\
0 & 0 & e \frac{(h-1)\left(1-\gamma_{1}\right)-\alpha_{1}}{\alpha_{1}-(h-1)}
\end{array}\right]
$$

The eigen values of the matrix $E_{1}$ are

$\lambda_{1}=r(h-1), \lambda_{2}=s$ and $\lambda_{3}=e \frac{(h-1)\left(1-\gamma_{1}\right)-\alpha_{1}}{\alpha_{1}-(h-1)}$.

If $h<1$ and $0<\gamma_{1}<1$ clearly two of the eigen vales are negative and one will be positive. Thus $E_{1}$ is stable manifold $x-z$ direction and unstable manifold in $y$ direction.

This completes the proof.

Theorem 4. The equilibrium point $E_{2}$ is locally asymptotically stable if $h>1$ and $\gamma_{2}<\alpha_{2}+1$ otherwise unstable in $x-z$ direction and stable in $y$ direction if $h<1$ and $\gamma_{2}>\alpha_{2}+1$.

Proof. The variational matrix for the equilibrium point at $E_{2}\left(0, \frac{1}{\alpha_{2}}, 0\right)$ is

$$
E_{2}=\left[\begin{array}{ccc}
r(1-h) & 0 & 0 \\
0 & -s & \frac{-\beta_{2} s}{1+\alpha_{2}} \\
0 & 0 & \frac{e \gamma_{2}}{1+\alpha_{2}}-e
\end{array}\right]
$$

The eigenvalues of the matrix $E_{2}$ are $\lambda_{1}=r(1-h), \lambda_{2}=-s$ and $\lambda_{3}=\frac{e \gamma_{2}}{1+\alpha_{2}}-e$.

If $h>1$ and $\gamma_{2}<\alpha_{2}+1$ in this case all the eigenvalues are negative. This shows the equilibrium $E_{2}$ of the system is locally asymptotically stable.

Suppose if $h<1$ and $\gamma_{2}>\alpha_{2}+1$ in this case two of the eigen values are positive and one of them will be negative. Hence it is unstable in $x-z$ direction and stable in $y$ direction.

This completes the proof.

Theorem 5. The equilibrium point $E_{3}$ is locally asymptotically stable if $h<1,0<\gamma_{1}<1$ and $\gamma_{2}<\alpha_{2}+1$.

Proof. The variational matrix for the equilibrium point at $E_{3}(\tilde{x}, \tilde{y}, 0)$

$$
E_{3}=\left[\begin{array}{ccc}
r-2 \alpha_{1} r \tilde{x}-h r & 0 & \frac{-\beta_{1} r \tilde{x}}{1+\tilde{x}} \\
0 & s-2 \alpha_{2} s \tilde{y} & \frac{-\beta_{2} s \tilde{y}(1+\tilde{y})}{(1+\tilde{y})^{2}} \\
0 & 0 & -e+\frac{\gamma_{1} e \tilde{x}}{(1+\tilde{x})}+\frac{\gamma_{2} e y(1+\tilde{y})}{(1+\tilde{y})^{2}}
\end{array}\right]
$$


Put $\tilde{x}=\frac{1-h}{\alpha_{1}}, \tilde{y}=\frac{1}{\alpha_{2}}$ then

$$
E_{3}=\left[\begin{array}{ccc}
-r(1-h) & 0 & \frac{\beta_{1} r(h-1)}{\alpha_{1}-(h-1)} \\
0 & -s & \frac{-\beta_{2} s}{1+\alpha_{2}} \\
0 & 0 & e\left[\frac{\gamma_{2}-\left(\alpha_{2}+1\right)}{\left(\alpha_{2}+1\right)}+\frac{\gamma_{1}(1-h)}{\alpha_{1}+(1-h)}\right]
\end{array}\right]
$$

The eigen values of the matrix $E_{3}$ are $-r(h-1),-s$ and $e\left[\frac{\gamma_{2}-\left(\alpha_{2}+1\right)}{\left(\alpha_{2}+1\right)}+\frac{\gamma_{1}(1-h)}{\alpha_{1}+(1-h)}\right]$.

All the eigenvalues are negative if $h<1,0<\gamma_{1}<1$ and $\gamma_{2}<\alpha_{2}+1$. This shows the equilibrium point $E_{3}$ of the system is locally asymptotically stable.

This completes the proof.

Theorem 6. The equilibrium point $E_{4}$ is locally asymptotically stable if $\alpha_{1}<(1-h)\left(\gamma_{1}-1\right)<2 \alpha_{1}$ and $\gamma_{1}>1$.

Proof. The variational matrix for the equilibrium point at $E_{4}(\bar{x}, 0, \bar{z})$ is

$$
E_{4}=\left[\begin{array}{ccc}
A_{1}^{*} & 0 & \frac{-\beta_{1} r \bar{x}}{(1+\bar{x})} \\
0 & B_{1}^{*} & 0 \\
\frac{\gamma_{1} e \bar{z}}{(1+\bar{x})^{2}} & \frac{\gamma_{2} e \bar{z}}{(1+\bar{z})} & C_{1}^{*}
\end{array}\right]
$$

Where

$$
A_{1}^{*}=r-2 \alpha_{1} r \bar{x}-\frac{\beta_{1} r \bar{z}}{(1+\bar{x})^{2}}, B_{1}^{*}=s-\frac{\beta_{2} s \bar{z}}{(1+\bar{z})^{2}} \text { and } C_{1}^{*}=-e+\frac{\gamma_{1} e \bar{x}}{(1+\bar{x})}
$$

Here $\bar{x}=\frac{1}{\gamma_{1}-1}$ and $\bar{z}=\frac{\gamma_{1}}{\left(\gamma_{1}-1\right)^{2} \beta_{1}}\left[(1-h)\left(\gamma_{1}-1\right)-\alpha_{1}\right]$

The eigen values of the matrix $E_{4}$ are negative real parts if $A_{1}^{*}<0$ and $B_{1}^{*}<0$ and $C_{1}^{*}<0$. By simple calculation we obtain the condition $\alpha_{1}<(1-h)\left(\gamma_{1}-1\right)<2 \alpha_{1}$ and $\gamma_{1}>1$.

Therefore the equilibrium point $E_{4}$ of the system is locally asymptotically stable.

This completes the proof.

Theorem 7. The equilibrium point $E_{5}$ is locally asymptotically stable if $h>1$ and $\gamma_{2}>1$.

Proof. The variational matrix for the equilibrium point at $E_{5}(0, \hat{y}, \hat{z})$ gives

$$
E_{5}=\left[\begin{array}{ccc}
A_{2}^{*} & 0 & 0 \\
0 & B_{2}^{*} & \frac{-\beta_{2} s \hat{y}(1+\hat{y})}{(1+\hat{y}+\hat{z})^{2}} \\
\frac{\gamma_{1} e \hat{z}}{1} & \frac{\gamma_{2} e \hat{z}(1+\hat{z})}{(1+\hat{y}+\hat{z})^{2}} & C_{2}^{*}
\end{array}\right]
$$

Where $A_{2}^{*}=r-\frac{\beta_{1} r \hat{z}}{1}-h r, B_{2}^{*}=s-2 \alpha_{2} s \hat{y}-\frac{\beta_{2} s \hat{z}}{(1+\hat{y}+\hat{z})^{2}}$ and $C_{2}^{*}=-e+\frac{\gamma_{2} e \hat{y}(1+\hat{y})}{(1+\hat{y}+\hat{z})^{2}}$

Here $\hat{y}=\frac{-\left(\beta_{2} \gamma_{2}-\gamma_{2}-\beta_{2}\right)+\sqrt{\left(\beta_{2} \gamma_{2}-\gamma_{2}-\beta_{2}\right)^{2}+4 \beta_{2} \gamma_{2} \alpha_{2}}}{2 \gamma_{2} \alpha_{2}}$,

$\hat{z}=\hat{y}\left(\gamma_{2}-1\right)-1$

The eigen values of the matrix $E_{5}$ are negative real parts if $A_{2}^{*}<0, B_{2}^{*}<0$ and $C_{2}^{*}<0$.

By simple calculation, we obtain the condition $h>1$ and $\gamma_{2}>1$.

Therefore the equilibrium point $E_{5}$ of the system is locally asymptotically stable in the $x-y-z$ direction.

This completes the proof. 
Theorem 8. The equilibrium point $E_{6}\left(x^{*}, y^{*}, z^{*}\right)$ is locally asymptotically stable if and only if the inequalities of (28) satisfied.

Proof. The variational matrix at the equilibrium point $E_{6}\left(x^{*}, y^{*}, z^{*}\right)$

Where

$$
E_{6}=\left[\begin{array}{ccc}
a_{11} & 0 & a_{13} \\
0 & a_{22} & a_{23} \\
a_{31} & a_{32} & a_{33}
\end{array}\right]
$$

$a_{11}=r-2 \alpha_{1} r x^{*}-\frac{\beta_{1} r z^{*}}{\left(1+x^{*}\right)^{2}}-h r, a_{13}=\frac{-\beta_{1} r x^{*}}{1+x^{*}}, a_{22}=s-2 \alpha_{2} s y^{*}-\frac{\beta_{2} s z^{*}\left(1+z^{*}\right)}{\left(1+y^{*}+z^{*}\right)^{2}}$

$a_{23}=\frac{-\beta_{2} s y^{*}\left(1+y^{*}\right)}{\left(1+y^{*}+z^{*}\right)^{2}}, a_{31}=\frac{\gamma_{1} e z^{*}}{\left(1+x^{*}\right)^{2}}, a_{32}=\frac{\gamma_{2} e z^{*}\left(1+z^{*}\right)}{\left(1+y^{*}+z^{*}\right)^{2}}, a_{33}=-e+\frac{\gamma_{1} e x^{*}}{\left(1+x^{*}\right)}+\frac{\gamma_{2} e y^{*}\left(1+y^{*}\right)}{\left(1+y^{*}+z^{*}\right)^{2}}$

Then the corresponding characteristic equation becomes

$$
\lambda^{3}+A_{1} \lambda^{2}+A_{2} \lambda+A_{3}=0
$$

Where

$$
\begin{aligned}
& A_{1}=-\left(a_{11}+a_{22}+a_{33}\right) \\
& =\left[(e+r(h-1)-s)+\left(2 \alpha_{1} r x^{*}+2 \alpha_{2} s y^{*}+\frac{\beta_{1} r z^{*}}{\left(1+x^{*}\right)^{2}}+\frac{\beta_{2} s z^{*}\left(1+z^{*}\right)}{\left(1+y^{*}+z^{*}\right)^{2}}\right)\right. \\
& \left.-\left(\frac{\gamma_{1} e x^{*}}{\left(1+x^{*}\right)}+\frac{\gamma_{2} e y^{*}\left(1+y^{*}\right)}{\left(1+y^{*}+z^{*}\right)^{2}}\right)\right] \\
& A_{22}=a_{22} a_{33}-a_{23} a_{32}+a_{11} a_{22}+a_{11} a_{33}-a_{13} a_{31} \\
& =\left[\left(s-2 \alpha_{2} s y^{*}-\frac{\beta_{2} s z^{*}\left(1+z^{*}\right)}{\left(1+y^{*}+z^{*}\right)^{2}}\right)\left(-e+\frac{\gamma_{1} e x^{*}}{\left(1+x^{*}\right)}+\frac{\gamma_{2} e y^{*}\left(1+y^{*}\right)}{\left(1+y^{*}+z^{*}\right)^{2}}\right)\right] \\
& +\left[\frac{\beta_{2} s y^{*}\left(1+y^{*}\right)}{\left(1+y^{*}+z^{*}\right)^{4}} \cdot \gamma_{2} e z^{*}\left(1+z^{*}\right)\right]+\left[\left(r-2 \alpha_{1} r x^{*}-\frac{\beta_{1} r z^{*}}{\left(1+x^{*}\right)^{2}}-h r\right)\right. \\
& \left.\cdot\left(s-2 \alpha_{2} s y^{*}-\frac{\beta_{2} s z^{*}\left(1+z^{*}\right)}{\left(1+y^{*}+z^{*}\right)^{2}}\right)\right]+\left[\left(r-2 \alpha_{1} r x^{*}-\frac{\beta_{1} r z^{*}}{\left(1+x^{*}\right)^{2}}-h r\right)\right. \\
& \left.\cdot\left(-e+\frac{\gamma_{1} e x^{*}}{\left(1+x^{*}\right)}+\frac{\gamma_{2} e y^{*}\left(1+y^{*}\right)}{\left(1+y^{*}+z^{*}\right)^{2}}\right)\right]+\left[\left(\frac{\beta_{1} r x^{*} \cdot \gamma_{1} e z^{*}}{\left(1+x^{*}\right)^{3}}\right)\right] \\
& A_{3}=\operatorname{det}\left(E^{*}\right) \\
& =a_{11} a_{32} a_{23}-a_{11} a_{22} a_{33}+a_{13} a_{22} a_{31} \\
& =-\left[r-2 \alpha_{1} r x^{*}-\frac{\beta_{1} r z^{*}}{\left(1+x^{*}\right)^{2}}-h r\right] \cdot\left[\left(s-2 \alpha_{2} s y^{*}-\frac{\beta_{2} s z^{*}\left(1+z^{*}\right)}{\left(1+y^{*}+z^{*}\right)^{2}}\right)\right. \\
& \left.\cdot\left(-e+\frac{\gamma_{1} e x^{*}}{\left(1+x^{*}\right)}+\frac{\gamma_{2} e y^{*}\left(1+y^{*}\right)}{\left(1+y^{*}+z^{*}\right)^{2}}\right)\right]-\left[r-2 \alpha_{1} r x^{*}-\frac{\beta_{1} r z^{*}}{\left(1+x^{*}\right)^{2}}-h r\right] \\
& \cdot\left[\frac{\beta_{2} s y^{*}\left(1+y^{*}\right)}{\left(1+y^{*}+z^{*}\right)^{4}} \cdot \gamma_{2} e z^{*}\left(1+z^{*}\right)\right]+\left[\frac{\left(\beta_{1} r x^{*}\right) \cdot \gamma_{1} e z^{*}}{\left(1+x^{*}\right)^{3}}\right] \cdot\left[s-2 \alpha_{2} s y^{*}-\frac{\beta_{2} s z\left(1+z^{*}\right)}{\left(1+y^{*}+z^{*}\right)^{2}}\right]
\end{aligned}
$$

Therefore an application of Routh-Hurwitz criterion shows that

$$
a_{11}<0, a_{22}<0
$$

Then the following conditions are satisfied

$$
A_{1}>0, A_{3}>0 \text { and } A_{1} A_{2}-A_{3}>0
$$

Hence the positive equilibrium point $E_{6}\left(x^{*}, y^{*}, z^{*}\right)$ is asymptotically stable.

This completes the proof. 


\section{Global Stability and Persistance}

Here we show the Global Stability Analysis of the system (2) by using Lasalle's invariance principle and suitable Lyapunov function.

\section{Global Stability of $E_{2}$}

We shall modify the system of equation (2) into a linear system to study the global stability of $E_{2}$ by considering a suitable Lyapunov function at the equilibrium point.

By letting

$x=x^{*}+x_{1} ; \quad y=y^{*}+y_{1} ; \quad z=z^{*}+z_{1}$

Where $x_{1}, y_{1}, z_{1}$ are small perturbations about $x^{*}, y^{*}, z^{*}$ respectively.

The linear system of equation (2) is

$$
\begin{aligned}
\frac{d x_{1}}{d t} & =\left[-\alpha_{1} r x^{*}+\frac{\beta_{1} r x^{*} z^{*}}{\left(1+x^{*}\right)^{2}}\right] x_{1}+\left[\frac{-\beta_{1} r x^{*}}{\left(1+x^{*}\right)}\right] z_{1} \\
\frac{d y_{1}}{d t} & =\left[-\alpha_{2} s y^{*}+\frac{\beta_{2} s y^{*} z^{*}}{\left(1+y^{*}+z^{*}\right)^{2}}\right] y_{1}+\left[\frac{-\beta_{2} s y^{*}\left(1+y^{*}\right)}{\left(1+y^{*}+z^{*}\right)^{2}}\right] z_{1} \\
\frac{d z_{1}}{d t} & =\left[\frac{\gamma_{1} e^{*} z^{*}}{\left(1+x^{*}\right)^{2}}\right] x_{1}+\left[\frac{\gamma_{2} e z^{*}\left(1+z^{*}\right)}{\left(1+y^{*}+z^{*}\right)^{2}}\right] y_{1}+\left[\frac{-\gamma_{2} e y^{*} z^{*}}{\left(1+y^{*}+z^{*}\right)^{2}}\right] z_{1}
\end{aligned}
$$

Considering a suitable Lyapunov function

$$
V\left(x_{1}, y_{1}, z_{1}\right)=\frac{x_{1}^{2}}{2}+\frac{y_{1}^{2}}{2 y^{*}}+\frac{z_{1}^{2}}{2}
$$

where $y^{*}$ is element of the equilibrium point $E_{2}$.

We note that $V\left(x_{1}, y_{1}, z_{1}\right)$ is a positive definite function.

Differentiate (30) with respect to $t$, we get

$$
\dot{V}\left(\dot{x}_{1}, \dot{y}_{1}, \dot{z}_{1}\right)=x_{1} \dot{x}_{1}+\frac{y_{1} \dot{y}_{1}}{y^{*}}+z_{1} \dot{z}_{1}
$$

Substituting (29) into (31), we get

$$
\begin{aligned}
\dot{V}\left(\dot{x}_{1}, \dot{y}_{1}, \dot{z}_{1}\right) & =x_{1}\left[\left[-\alpha_{1} r x^{*}+\frac{\beta_{1} r x^{*} z^{*}}{\left(1+x^{*}\right)^{2}}\right] x_{1}+\left[\frac{-\beta_{1} r x^{*}}{\left(1+x^{*}\right)}\right] z_{1}\right] \\
& +y_{1}\left[\left[-\alpha_{2} s+\frac{\beta_{2} s z^{*}}{\left(1+y^{*}+z^{*}\right)^{2}}\right] y_{1}+\left[\frac{-\beta_{2} s\left(1+y^{*}\right)}{\left(1+y^{*}+z^{*}\right)^{2}}\right] z_{1}\right] \\
& +z_{1}\left[\left[\frac{\gamma_{1} e^{*} z^{*}}{\left(1+x^{*}\right)^{2}}\right] x_{1}+\left[\frac{\gamma_{2} e z^{*}\left(1+z^{*}\right)}{\left(1+y^{*}+z^{*}\right)^{2}}\right] y_{1}+\left[\frac{-\gamma_{2} e y^{*} z^{*}}{\left(1+y^{*}+z^{*}\right)^{2}}\right] z_{1}\right]
\end{aligned}
$$

In the equilibrium point $E_{2}, x^{*}=0, z^{*}=0$

On simplification of (32) we have

$\dot{V}\left(\dot{x}_{1}, \dot{y}_{1}, \dot{z}_{1}\right)=\left[-\alpha_{2} s\right] y_{1}^{2}+\left[\frac{-\beta_{2} s}{\left(1+y^{*}\right)}\right] y_{1} z_{1}$

Which is negative semi definite.

Therefore $E_{2}\left(0, \frac{1}{\alpha_{2}}, 0\right)$ is Lyapunov stable.

Thus the set

$S=\left\{\left(x_{1}, y_{1}, z_{1}\right) / \dot{V}\left(\dot{x}_{1}, \dot{y}_{1}, \dot{z}_{1}\right)=0\right\}$ becomes $S=\left\{\left(x_{1}, y_{1}, z_{1}\right) / y_{1}=0\right\}$ when it contains only the trivial trajectory by LaSalle's invariance principle $E_{2}\left(0, \frac{1}{\alpha_{2}}, 0\right)$ is globally asymptotically stable. 
Theorem 9. The interior equilibrium $E_{3}$ is globally asymptotically stable in the interior of the quadrant of the $x-y$ plane.

Proof. Let $H_{1}(x, y)=\frac{1}{x y}$

Clearly $H_{1}(x, y)$ is positive in the interior of the positive quadrant of $x-y$ plane.

Let

$h_{1}(x, y)=r x\left(1-\alpha_{1} x-h\right)$

$h_{2}(x, y)=s y\left(1-\alpha_{2} y\right)$

Then $\Delta(x, y)=\frac{\partial}{\partial x}\left(h_{1} H_{1}\right)+\frac{\partial}{\partial y}\left(h_{2} H_{1}\right)$

$=\frac{-r \alpha_{1}}{y}-\frac{s \alpha_{2}}{x}$

$<0$

Using Bendixson-Dulac criteria, we note that $\Delta(x, y)$ remains the same sign and is not identically zero in the interior of the positive quadrant of the $x-y$ plane.

This completes the proof.

We shall now prove that $E_{4}$ is globally asymptotically stable.

Theorem 10. The interior equilibrium $E_{4}$ is globally asymptotically stable in the interior of the quadrant of the $x-z$ plane.

Proof. Let $H_{2}(x, z)=\frac{1}{x z^{2}}$

Clearly $\mathrm{H}_{2}(x, z)$ is positive in the interior of the positive quadrant of $x-z$ plane.

Let

$h_{1}(x, z)=r x\left[\left(1-\alpha_{1} x-\frac{\beta_{1} z}{1+x}-h\right)\right]$

$h_{2}(x, z)=e z\left[-1+\frac{\gamma_{1} x}{1+x}\right]$

Then $\Delta(x, z)=\frac{\partial}{\partial x}\left(h_{1} H_{2}\right)+\frac{\partial}{\partial z}\left(h_{2} H_{2}\right)$

$=-\frac{1}{z^{2}}\left[\frac{-e+r \alpha_{1} x}{x}+\frac{-r \beta_{1} z+e \gamma_{1}+e \gamma_{1} x}{(1+x)^{2}}\right]$

$<0$

Using Bendixson-Dulac criteria, we note that $\Delta(x, z)$ remains the same sign and is not identically zero in the interior of the positive quadrant of the $x-z$ plane.

This completes the proof.

We shall now prove that $E_{5}$ is globally asymptotically stable.

Theorem 11. The interior equilibrium $E_{5}$ is globally asymptotically stable in the interior of the quadrant of the $y-z$ plane.

Proof. Let $H_{3}(y, z)=\frac{1}{y z}$

Clearly $H_{3}(y, z)$ is positive in the interior of the positive quadrant of $y-z$ plane. 
Let

$h_{1}(y, z)=s y\left(1-\alpha_{2} y-\frac{\beta_{2} z}{1+y+z}\right)$

$h_{2}(y, z)=e z\left[-1+\frac{\gamma_{2} y}{1+y+z}\right]$

Then $\Delta(y, z)=\frac{\partial}{\partial y}\left(h_{1} H_{3}\right)+\frac{\partial}{\partial z}\left(h_{2} H_{3}\right)$

$=-\frac{s \alpha_{2}}{z}-\left(\frac{e \gamma_{2}-s \beta_{2}}{(1+y+z)^{2}}\right)$

$<0$

Using Bendixson-Dulac criteria, we note that $\Delta(y, z)$ remains the same sign and is not identically zero in the interior of the positive quadrant of the $y-z$ plane.

This completes the proof.

We shall now prove that $E_{6}$ is globally asymptotically stable.

Theorem 12. The co-existence equilibrium point $E_{6}\left(x^{*}, y^{*}, z^{*}\right)$ is globally asymptotically stable with respect to all solutions initiating in the interior of $B$ satisfy the following conditions

$$
\begin{aligned}
& z^{*}<\min \left(\frac{\beta_{1} R_{1}}{\gamma_{1}}, \frac{\beta_{2} R_{2}}{\gamma_{2}}\right) \\
& x^{*}=\frac{\gamma_{1}-\beta_{1}}{\beta_{1}} \\
& y^{*}<\frac{\gamma_{2}\left(\beta_{1}+\alpha_{1} R_{1}\right)-\beta_{1} \beta_{2}}{\beta_{1} \beta_{2}}, \gamma_{1}>\beta_{1}, \gamma_{2}>\beta_{2}
\end{aligned}
$$

Proof. The proof can be reached by using Lyapunov stability theorem which gives sufficient condition.

Now let us consider a positive definite function $V(x, y, z)$ such that

$$
\begin{aligned}
V(x, y, z)= & \frac{1}{r}\left[\left(x-x^{*}\right)-x^{*} \ln \left(\frac{x}{x^{*}}\right)\right]+\frac{1}{s}\left[\left(y-y^{*}\right)\right. \\
& \left.-y^{*} \ln \left(\frac{y}{y^{*}}\right)\right]+\frac{1}{e}\left[\left(z-z^{*}\right)-z^{*} \ln \left(\frac{z}{z^{*}}\right)\right]
\end{aligned}
$$

in the interior of the positive octant.

Differentiate (34) with respect to time $t$ we get

$$
\dot{V}=\left(x-x^{*}\right) \frac{\dot{x}}{r x}+\left(y-y^{*}\right) \frac{\dot{y}}{s y}+\left(z-z^{*}\right) \frac{\dot{z}}{e z}
$$

Using system of equation (2) into (35)

$$
\begin{aligned}
\dot{V}= & \left(x-x^{*}\right)\left[1-\alpha_{1} x-\frac{\beta_{1} z}{1+x}-h\right]+\left(y-y^{*}\right)\left[1-\alpha_{2} y-\frac{\beta_{2} z}{1+y+z}\right] \\
& +\left(z-z^{*}\right)\left[\frac{\gamma_{1} x}{1+x}+\frac{\gamma_{2} y}{1+y+z}-1\right]
\end{aligned}
$$

After simple calculation we have

$\dot{V}=-\left(x-x^{*}\right)^{2}\left(\alpha_{1}-\frac{\beta_{1} z^{*}}{(1+x)\left(1+x^{*}\right)}\right)-\left(x-x^{*}\right)\left(z-z^{*}\right)\left(\frac{\beta_{1}\left(1+x^{*}\right)-\gamma_{1}}{(1+x)\left(1+x^{*}\right)}\right)$ 


$$
\begin{aligned}
& -\left(y-y^{*}\right)^{2}\left(\alpha_{2}-\frac{\beta_{2} z^{*}}{(1+y+z)\left(1+y^{*}+z^{*}\right)}\right)-\left(y-y^{*}\right)\left(z-z^{*}\right)\left(\frac{\beta_{2}\left(1+y^{*}\right)-\gamma_{2}\left(1+z^{*}\right)}{(1+y+z)\left(1+y^{*}+z^{*}\right)}\right) \\
& -\left(z-z^{*}\right)^{2} \frac{\gamma_{2} y^{*}}{(1+y+z)\left(1+y^{*}+z^{*}\right)}
\end{aligned}
$$

The above equation can be written as

$\dot{V}=-\left[b_{11}\left(x-x^{*}\right)^{2}+b_{13}\left(x-x^{*}\right)\left(z-z^{*}\right)+b_{22}\left(y-y^{*}\right)^{2}+b_{23}\left(y-y^{*}\right)\left(z-z^{*}\right)+b_{33}\left(z-z^{*}\right)^{2}\right]$

Where

$b_{11}=\left(\alpha_{1}-\frac{\beta_{1} z^{*}}{R_{1}}\right), b_{13}=\left(\frac{\beta_{1}\left(1+x^{*}\right)-\gamma_{1}}{R_{1}}\right), b_{22}=\left(\alpha_{2}-\frac{\beta_{2} z^{*}}{R_{2}}\right)$,

$b_{23}=\left(\frac{\beta_{2}\left(1+y^{*}\right)-\gamma_{2}\left(1+z^{*}\right)}{R_{2}}\right), \quad b_{33}=\frac{\gamma_{2} y^{*}}{R_{2}}$

Here $R_{1}=(1+x)\left(1+x^{*}\right), R_{2}=(1+y+z)\left(1+y^{*}+z^{*}\right)$

Then the sufficient condition that $\dot{V}$ to be negative definite is

$b_{11}>0, b_{12}^{2}<4 b_{11} b_{22}$.

Hence $V$ is a Lyapunov function with respect to $E_{6}\left(x^{*}, y^{*}, z^{*}\right)$.

Therefore co-existence equilibrium point $E_{6}$ is globally asymptotically stable.

In the next theorem, we show that system (2) is uniformly persistent. By the permanence or persistence of a system, we mean that all the species are present and none of them will go to extinction.

Theorem 13. Let the hypotheses of Theorems 9, 10 and 11 hold and then the system (2) is uniformly persistent if following inequalities hold

(i) $s(1+\bar{z})^{2}>\beta_{2} s \bar{z}$

(ii) $r>\left(\beta_{1} r \hat{z}+h r\right)$

Proof. We prove this theorem by method of average Lyapunov function.

Let the average Lyapunov function for system (2) be

$$
\sigma(X)=x^{p} y^{q} z^{r}
$$

where $p, q$ and $r$ are positive constants.

Clearly $\sigma(X)$ is a non-negative function defined in $D$ of $R_{+}^{3}$

where

$D \equiv\left\{(x, y, z), x>0, y>0, z>0, \alpha_{2}(1+y+z)^{2}+\frac{\left(e \gamma_{2}-s \beta_{2}\right) z}{s}>0\right\}$

Then we have

$\psi(X)=\frac{\dot{\sigma}(X)}{\sigma(X)}$

$=p \frac{\dot{x}}{x}+q \frac{\dot{y}}{y}+r \frac{\dot{z}}{z}$

$=p\left[r\left(1-\alpha_{1} x-\frac{\beta_{1} z}{1+x}-h\right)\right]+q\left[s\left(1-\alpha_{2} y-\frac{\beta_{2} z}{1+y+z}\right)\right]+r\left[e\left(\frac{\gamma_{1} x}{1+x}+\frac{\gamma_{2} y}{1+y+z}-1\right)\right]$

Further, hypotheses of Theorems 9, 10 and 11 implies that there are no-periodic orbits in the interior of positive quadrant of $x-y$ plane, $x-z$ plane and $y-z$ plane.

Thus to prove that uniform persistence of the system, it is enough to show $\psi(x)>0$ in $R_{+}^{3}$ for a suitable choice of $p, q, r>0$. 


$$
\begin{aligned}
& \psi\left(E_{0}\right)=p[r(1-h)]+q s-\gamma e>0 \\
& \psi\left(E_{1}\right)=q s+r\left[\frac{e(h-1)\left(1-\gamma_{1}\right)-\alpha_{1}}{\alpha_{1}-(h-1)}\right]>0 \\
& \psi\left(E_{2}\right)=p(r(1-h))+r\left[\frac{e \gamma_{2}}{1+\alpha_{2}}-e\right]>0 \\
& \psi\left(E_{3}\right)=r\left[e\left[\frac{\gamma_{2}-\left(\alpha_{2}+1\right)}{\alpha_{2}+1}+\frac{\gamma_{1}(1-h)}{\alpha_{1}+(1-h)}\right]\right]>0 \\
& \psi\left(E_{4}\right)=q\left[s-\frac{\beta_{2} s \bar{z}}{(1+\bar{z})^{2}}\right]>0 \\
& \psi\left(E_{5}\right)=p\left[r-\beta_{1} \gamma \hat{z}-h r\right]>0
\end{aligned}
$$

We note that by increasing $p$ to sufficiently large value $\psi\left(E_{0}\right)$ can be made positive. This completes the proof.

\section{Numerical Simulations}

Analytical studies become complete only with the numerical justification of the results. A qualitative analysis of the main features in the system is described by numerical simulations. Therefore, we assign some hypothetical data in order to verify the analytical result that has been obtained. The numerical experiments are conducted to examine the dynamical behavior of the system in three different parameter sets. It is obvious that changing the parameter value changes the numerical outcomes. So every different set of parameter gives unique results.

Let $R_{1}$ be the parameter set taken as

$$
\begin{aligned}
& r=3, s=2.5, K=50, L=30, a=1, b=0.04, c=0.001, \\
& \lambda_{1}=0.125, \lambda_{2}=0.5, e=0.5, d_{1}=0.001, d_{2}=1
\end{aligned}
$$

With the above parameter set, the system (1) has varying harvesting rate. If the intrinsic growth rate is higher than the harvesting rate, the predator population becomes extinct and the population density of one prey (prey 1) becomes low while that of the other becomes high (Refer Fig. 1 and 2). If the harvesting rate exceeds the intrinsic growth rate of the prey, the prey and predator becomes extinct. (Refer Fig.3). This implies that the harvesting rate plays a pre-dominant in the stability of system (1).

Let $R_{2}$ be the parameter set taken as

$$
\begin{aligned}
& r=2, s=1, K=50, L=30, a=1, b=0.04, c=0.001, \\
& \lambda_{1}=0.125, \lambda_{2}=0.5, d_{1}=0.001, d_{2}=1, H=0.5
\end{aligned}
$$

In the above parameter set at $e=0.7$, it is observed that stable spiral exists (see Fig. 4 and 5) which is globally asymptotically stable.

Let $R_{3}$ be the parameter set taken as

$$
\begin{aligned}
& r=2, s=1, K=50, L=30, a=1, b=0.04, c=0.001, \\
& \lambda_{2}=0.5, e=0.5, d_{1}=0.001, d_{2}=1, H=0.5
\end{aligned}
$$

The graphical results obtained from the parameter set $R_{3}$ infers that increasing the value of $\lambda_{1}$ will increase the population density of the predator and second prey but decrease the population density of first prey (Refer Fig. 6-7) and phase portrait of the system (Refer Fig.8). 


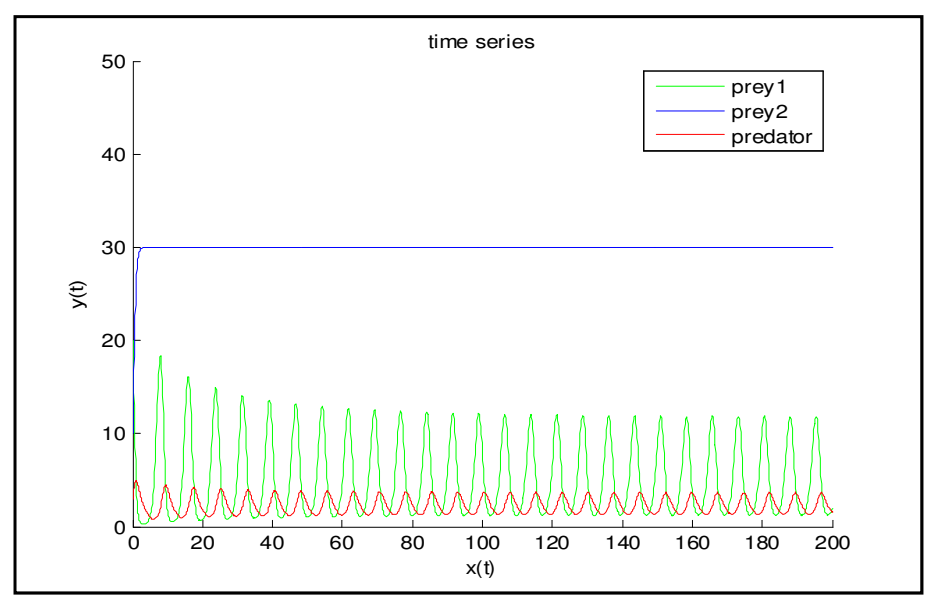

Fig.1. Numerical solution of system (1) at $\mathrm{H}=0.75$

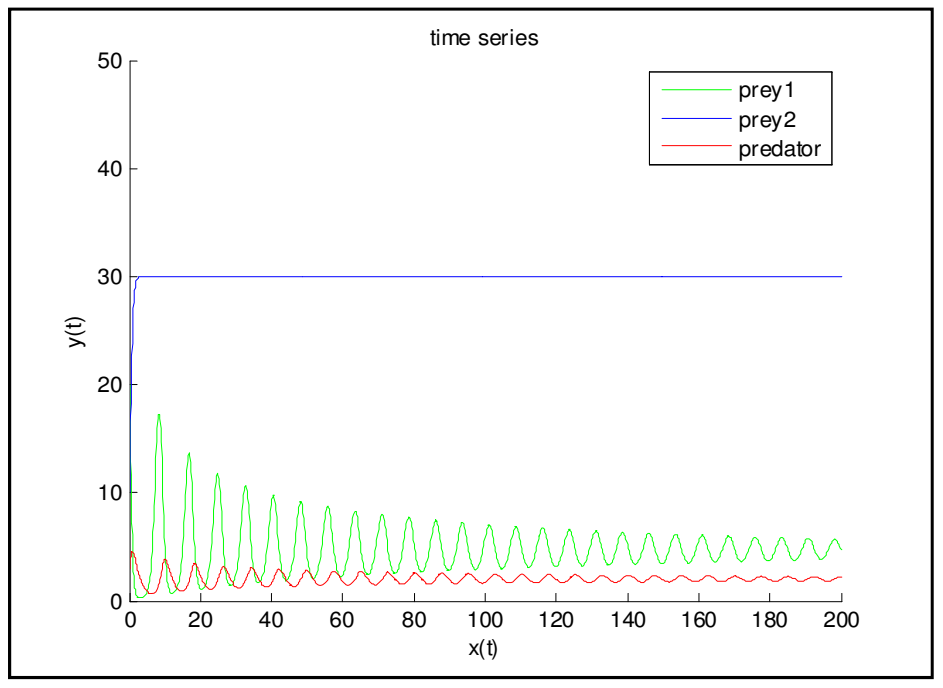

Fig.2. Numerical solution of system (1) at $\mathrm{H}=1$

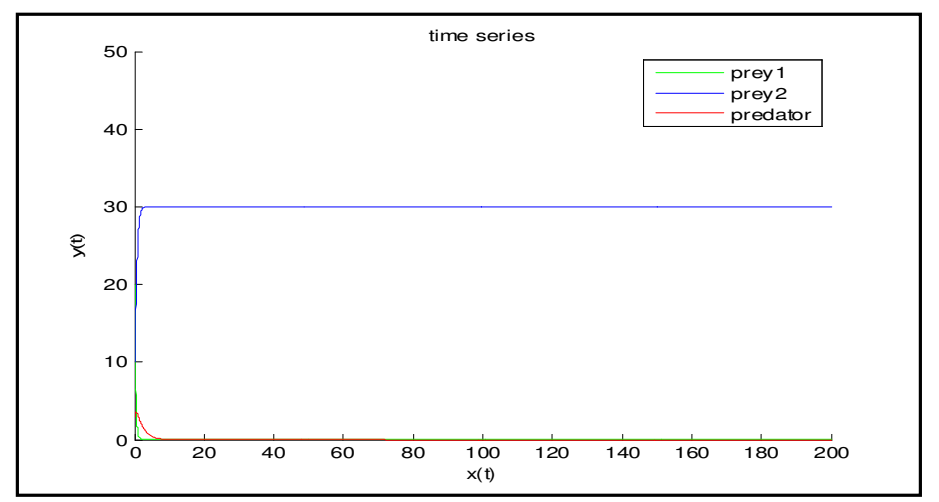

Fig.3.Numerical solution of system (1) at $\mathrm{H}=3$ 


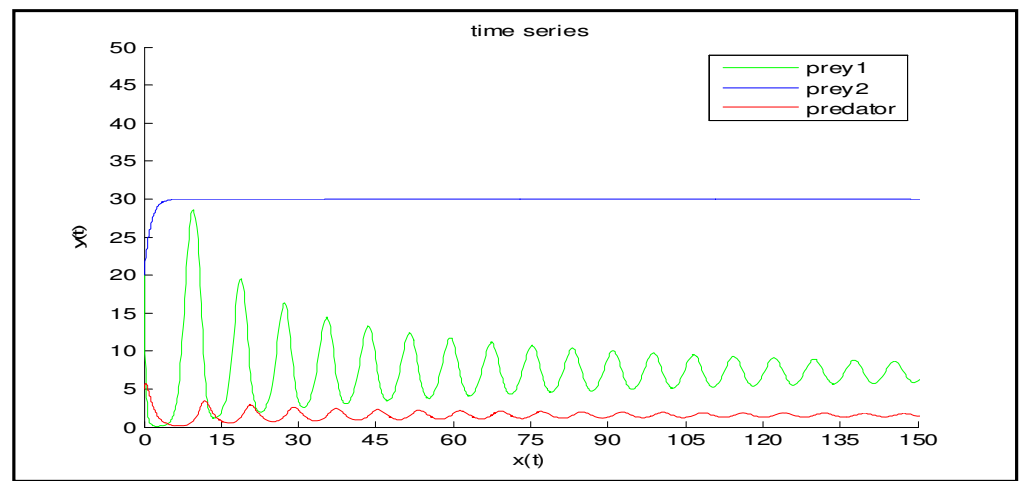

Fig.4 Numerical solution of system (1) at $\mathrm{e}=0.7$

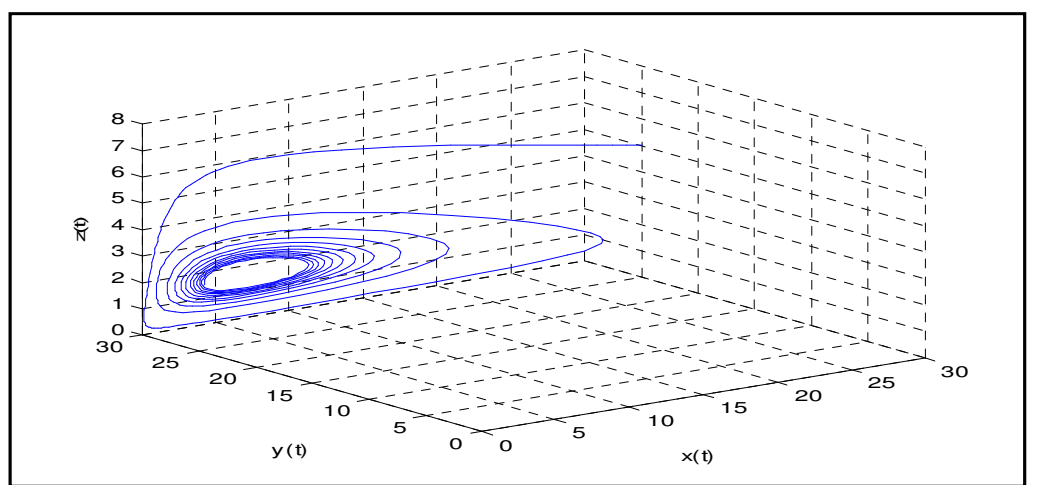

Fig.5 Phase portrait of system (1) at $\mathrm{e}=0.7$

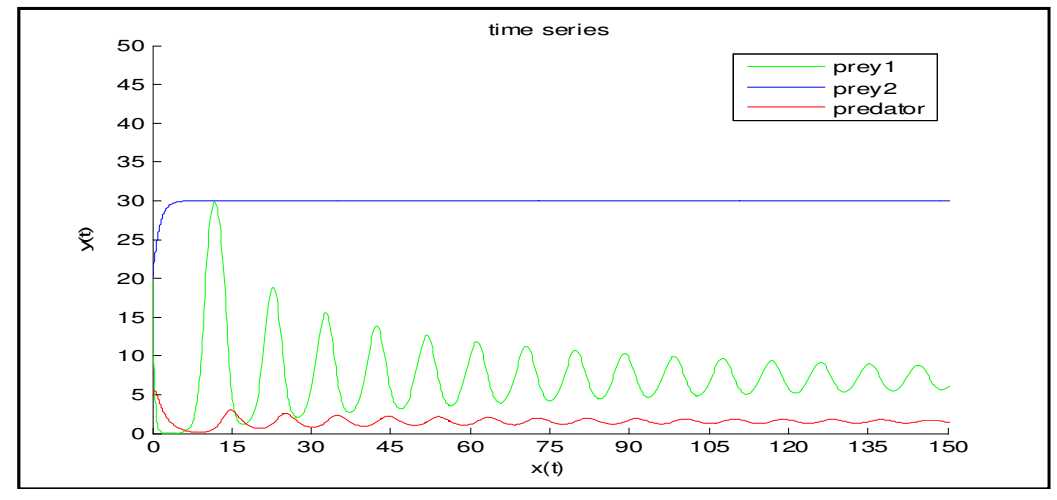

Fig.6 Numerical solution of system (1) at $\lambda_{1}=0.09$ 


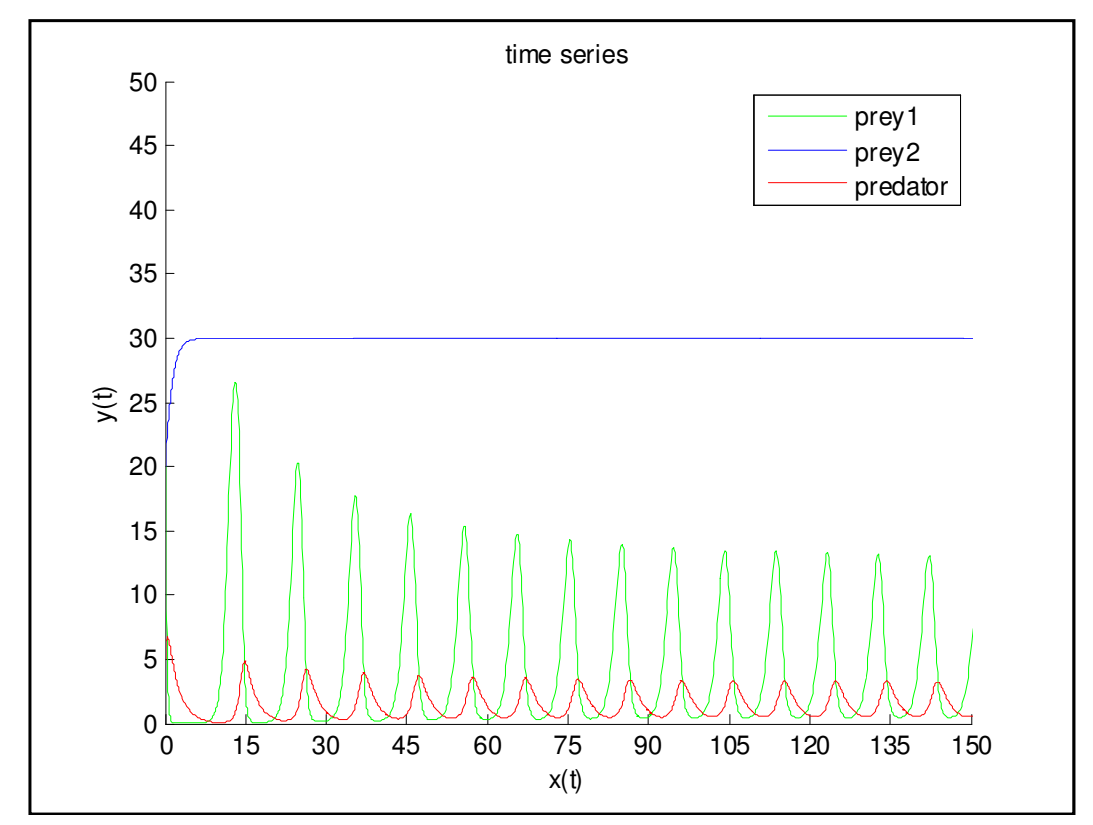

Fig.7 Numerical solution of system (1) at $\lambda_{1}=0.15$

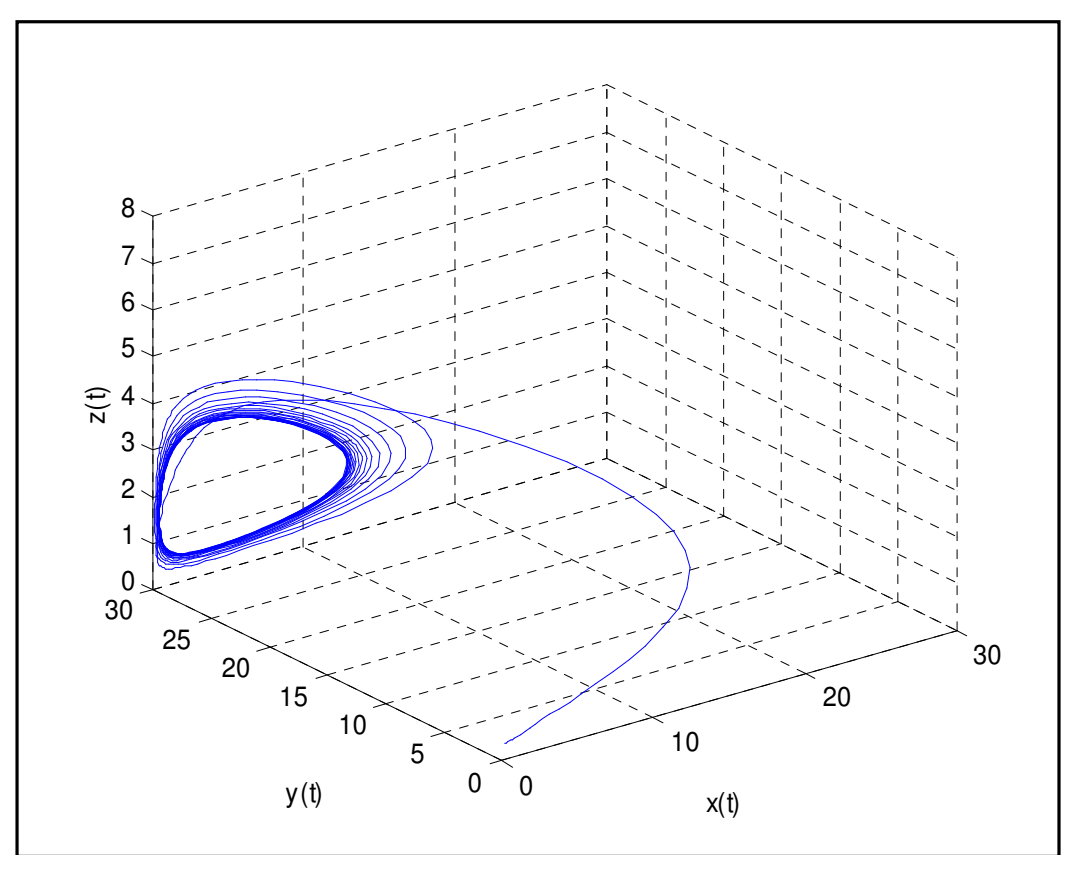

Fig. 8 Phase portrait of system (1) at $\lambda_{1}=0.15$ 


\section{Conclusion}

In this paper, we studied the dynamical behavior of a two prey and one predator system. In this model system predator exhibits holling type II functional response to one prey and Bedding ton-De angelis functional response to other. Such a different choice of functional response is useful, when handling time for one prey is negligble where as the predator needs sufficient handling time for other.

To examine the dynamical behaviour of the system, we have first discussed the existence of possible steady states and their local stability. Global stability of the system is shown by using Lyapunov stability theorem. The condition for the persistance in the system examined. Our Mathematical model shows that, using the parameter $H$ and $\lambda_{1}$ as control, it is possible to break stable state into unstable state. Also it is possible to keep the population level in finite time. More over the system is driven from limit cycle to stable spiral by varying natural death of predator $(e)$ and food conversion rate of predator $\left(\lambda_{1}\right)$ that are globally asymptotically stable.

\section{Acknowledgment}

The authors would like to extend their appreciation to the anonymous referees for their many helpful comments and suggestions which greatly improved the presentation of this paper.

\section{References}

[1] B. Dubey, R.K. Upadhyay, Persistence and extinction of one prey and two predator system, J. Nonlinear Analysis. Modelling and Control, Vol.9, No.4, (2004) 307-329.

[2] E.A. McGehee, N. Schutt, D.A. Vasquez and E. Peacock-Lopez, Bifurcations and temporal and spatial patterns of a modified Lotka-Volterra model, Int.J. Bifurcation Chaos Appl.Sci.Eng. 18 (2008) 2223-2248.

[3] S. Gakkar and R.K. Naji, Existence of chaos in two prey and one predator system. chaos, Solutions \& Fractals, 17(4) (2003) 639-649.

[4] S. Gakkar and R.K. Naji, Chaos in three species ratio dependent food chain. chaos, Solutions \& Fractals 14 (2002) 771-778.

[5] H.F. Huo, Z.P.Ma and C.Y.Liu, Persistence and Stability for a Generalized Leslie-Gower Model with Stage Structure and Dispersal, Hindawi Publishing Corporation Abstract and Applied Analysis, Article ID 135843 (2009), 17 pages.

[6] S.B. Hsu, T.W. Hwang and Y. Kuang, Rich dynamics of a ratio-dependent one prey-two predators model, J. Math. Biol., Vol. 43. (2001) 377-396.

[7] D. Kesh, A.K. Sarkar and A.B. Roy, Persistence of two prey-one predator system with ratiodependent predator influence, Math. Appl. Sci., Vol.23 (2000) 347-356.

[8] S. Kumar, S.K. Srivastava and P. Chingakham, Hopf bifuracation and stability analysis in a harvested one-predator-two-prey model, Appl. Math. Comput., Vol 129, No. 1, (2002) 107-118.

[9] M. Haque, A detailed study of the Beddington-DeAngelis predator-prey model, Math. Biosci. 234 (2011) 116.

[10] P. Lenzini and J. Rebaza, Non-constant predator harvesting on ratio-dependent prey-predator models, Appl. Math. Sci. (16)(2010) 791-803. 
[11] R.K. Naji and A.T. Balasim, Dynamical behavior of a three species food chain model with Beddington- DeAngelis functional response, Chaos, Solutions and Fractals, 32 (2007), 18531866.

[12] S. Gakkar and Brahampal Singh, The Dynamics of food web consisting of two preys and a harvesting predator, Chaos, Solutions and Fractals 34 (2007) 1346-1356.

[13] T.K. Kar and A. Batabyal, Persistence and Stability of a Two Prey One Predator System, International Journal of Engg. Sci. and Tech., 2(2) (2010) 174-190.

[14] D. Xiao and S. Ruan, Global dynamics of a ratio dependent predator-prey system, J. Math. Biol., 43(3) (2001) 268-290. 